\title{
Exploration of global gene expression in human liver steatosis by high-density oligonucleotide microarray
}

\author{
Franck Chiappini ${ }^{1,2}$, Alain Barrier ${ }^{3,4}$, Raphaël Saffroy ${ }^{1}$, Marie-Charlotte Domart ${ }^{1}$, \\ Nicolas Dagues ${ }^{2}$, Daniel Azoulay ${ }^{1,5}$, Mylène Sebagh ${ }^{1,6}$, Brigitte Franc ${ }^{7}$, \\ Stephan Chevalier ${ }^{2}$, Brigitte Debuire ${ }^{1}$, Sandrine Dudoit ${ }^{4}$ and Antoinette Lemoine ${ }^{1}$
}

\begin{abstract}
${ }^{1}$ Inserm 602, Service de Biochimie et Biologie Moléculaire, Hôpital Universitaire Paul Brousse, Université Paris XI, Villejuif Cedex, France; ${ }^{2}$ Pfizer, Centre de Recherche, Amboise, France; ${ }^{3}$ Service de Chirurgie Digestive, Hôpital Tenon, Inserm 444, Université Pierre et Marie Curie, Paris, France; ${ }^{4}$ Division of Biostatistics, University of California, Berkeley, CA, USA; ${ }^{5}$ Centre Hépato-Biliaire, Hôpital Universitaire Paul Brousse, Villejuif, France; ${ }^{6}$ Service d'Anatomie pathologique, Hôpital Universitaire Paul Brousse, Villejuif, France and ${ }^{7}$ Service d'Anatomie pathologique, Hôpital Ambroise Paré, Assistance Publique-Hôpitaux de Paris, Boulogne Billamcourt, France
\end{abstract}

\begin{abstract}
Understanding the molecular mechanisms underlying fatty liver disease (FLD) in humans is of major importance. We used high-density oligonucleotide microarrays $(22.3 \mathrm{~K})$ to assess the mechanisms responsible for the development of human liver steatosis. We compared global gene expression in normal $(n=9)$ and steatotic $(n=9)$ livers without histological signs of inflammation or fibrosis. A total of 34 additional human samples including normal $(n=11)$, steatosis $(n=11)$, HCV-related steatosis $(n=4)$ or steatohepatitis associated with alcohol consumption $(n=4)$ or obesity $(n=4)$ were used for immunohistochemistry or quantitative realtime PCR studies. With unsupervised classification (no gene selection), all steatotic liver samples clustered together. Using step-down maxT multiple testing procedure for controlling the Family-Wise Error-Rate at level $5 \%, 110$ cDNAs (100 over- and 10 underexpressed) were found to be differentially expressed in steatotic and normal livers. Of them were genes involved in mitochondrial phosphorylative and oxidative metabolism. The mean ratio of mitochondrial DNA to nuclear DNA content was higher in liver steatosis compared to normal liver biopsies $(1.12 \pm 0.14$ vs $0.67 \pm 0.10 ; P=0.01)$. An increased expression of genes involved in inflammation (IL-1R family, TGFB) was also observed and confirmed by quantitative RT-PCR or immunochemistry. In steatohepatitis, an increase of the protein expression of mitochondrial antigens, IL-1R1, IGF2 and TGFB1 was also observed, interleukin 1 receptor being always strongly expressed in steatohepatitis linked to alcohol or obesity. In conclusion, mitochondrial alterations play a major role in the development of steatosis per se. Activation of inflammatory pathways is present at a very early stage of steatosis, even if no morphological sign of inflammation is observed.

Laboratory Investigation (2006) 86, 154-165. doi:10.1038/labinvest.3700374; published online 12 December 2005
\end{abstract}

Keywords: steatosis; TGFB1; IL-1R; mitochondria; inflammation; gene expression profiling

Fatty liver disease (FLD) is a term used to describe a wide spectrum of clinical conditions that result in triglyceride accumulation (steatosis) and progressive necroinflammatory liver disease (steatohepatitis). Steatosis and steatohepatitis are often attributed

Correspondence: Dr A Lemoine, DPharm, PhD, Service de Biochimie et Biologie Moléculaire-INSERM 602, Hôpital Paul Brousse, 14 avenue Paul Vaillant Couturier, 94804 Villejuif Cedex, France.

E-mail: antoinette.lemoine@pbr.ap-hop-paris.fr

Received 2 May 2005; revised 21 October 2005; accepted 25 October 2005; published online 12 December 2005 to the effects of excessive alcohol consumption, obesity, diabetes and drugs (for reviews see Ried $^{1}$ and Diehl ${ }^{2}$ ). When the aetiology is not alcoholic, the disease is referred to as nonalcoholic steatohepatitis (NASH) or nonalcoholic fatty liver disease (NAFLD). The most common disorders associated with liver steatosis are insulin resistance and obesity. ${ }^{1,2}$ Recent epidemiological data showed that steatosis could affect a considerable number of subjects, as $30 \%$ of North American adults-nearly 59 million peoplewere obese in $2000 .^{3}$ Growing obesity rates are not confined to the US. Obesity is also related with other health risks including cardiovascular disease, 
diabetes, asthma, arthritis and cancer. ${ }^{4}$ Changes in liver structure due to lipid accumulation have deleterious effects as they increase the sensitivity of the liver to several types of injury, including injury due to drug toxicity and surgery. It was recently suggested that rather than being an 'innocent bystander', liver steatosis may be the 'guilty party' of the progression to cirrhosis ${ }^{5-7}$ and possibly in the development of hepatocellular carcinoma (HCC). ${ }^{8}$ This can result in end-stage liver disease requiring liver transplantation. ${ }^{9-11}$ Therefore, understanding the molecular mechanisms underlying FLD is of major importance. Mechanisms responsible for the development of steatosis, the hallmark feature of steatohepatitis, have been extensively studied in mouse models. However, because of difficulties of such studies in humans, little is known about the molecular and cellular pathways involved in humans. In fact, available evidence points to interspecies differences in the mechanisms involved. ${ }^{12}$

Most published studies have analysed only one or two genes or gene products in liver steatosis or NASH and have therefore not demonstrated the potential molecular diversity of this frequently seen liver histological feature. Recent advances in microarray technology and bioinformatics have revealed molecular heterogeneity based on genome-wide gene expression profiles. Recently published microarray data have demonstrated significant differences in the expression of genes important for maintaining mitochondrial function between NASH patients and healthy subjects. ${ }^{13}$ Here, we compared genome-wide mRNA levels in normal livers and in fatty livers without histological features of inflammation to improve our understanding of the physiopathogenesis of steatosis per se.

\section{Materials and methods}

\section{Patients and Tissue Samples}

In our institution, liver biopsies are systematically collected during graft harvesting to check the histological quality of the liver graft before orthotopic transplantation. From 72 consecutive liver biopsies, 18 were selected based on the following criteria: a stay in Intensive Care Unit for less than $24 \mathrm{~h}$, the absence of pathological features of inflammation and RNA preparation of good quality to perform microarrays. There were nine normal liver biopsies and nine steatotic liver biopsies; the characteristics of patients are detailed in Table 1 . Causes of death were accidental cerebral impact in seven cases and vascular cerebral failure in 11 cases. All the donors responded to the criteria for liver transplantation and results of liver function tests were within the accepted range for transplantation. Haemostasis parameters were normal and prothrombin time was greater than $70 \%$ in all cases. No infection with viruses such as hepatitis B virus (HBV), hepatitis C virus (HCV), human
Table 1 Characteristics of patients included in the gene profiling study

\begin{tabular}{lccc}
\hline & \multicolumn{3}{c}{ Patients } \\
\cline { 2 - 4 } & $\begin{array}{c}\text { Without } \\
\text { steatosis }(\mathrm{N}=9)\end{array}$ & $\begin{array}{c}\text { With steatosis } \\
(\mathrm{N}=9)\end{array}$ & $\mathrm{P}$ \\
\hline Age (years) & $38.5 \pm 19.2$ & $40.4 \pm 17.0$ & $\mathrm{NS}$ \\
Sex (M/F) & $7 / 2$ & $7 / 2$ & $\mathrm{NS}$ \\
BMI (kg/m $\left.{ }^{2}\right)$ & $22.7 \pm 3.0$ & $26.5 \pm 4.3$ & $<0.05$ \\
Weight of graft (g) & $1469 \pm 383$ & $1696 \pm 678$ & $\mathrm{NS}$ \\
Total cholesterol & $4.76 \pm 0.90$ & $4.45 \pm 1.23$ & $\mathrm{NS}$ \\
(mmol/l) & & & \\
Triglycerides & $1.71 \pm 0.47$ & $1.93 \pm 0.76$ & $\mathrm{NS}$ \\
(mmol/l) & $44 \pm 19$ & $33 \pm 20$ & $\mathrm{NS}$ \\
AST (U/l) & $33 \pm 16$ & $30 \pm 20$ & $\mathrm{NS}$ \\
ALT (U/l) & $25 \pm 18$ & $36 \pm 21$ & $\mathrm{NS}$ \\
GGT (U/l) & & \\
\hline
\end{tabular}

ALT, alanine aminotransferase; AST, aspartate aminotransferase; BMI, body mass index; GGT, gamma glutamyltransferase; NS, nonsignificant.

T-cell leukaemia lymphoma virus (HTLV)1 and HTLV2 was detected. Creatinin levels were below $100 \mathrm{mmol} / \mathrm{l}$ and none of the donors had diabetes. Height and weight were recorded for each donor and body mass index $\left(B M I=\right.$ weight $(\mathrm{kg}) /\left(\right.$ height $\left.(\mathrm{m})^{2}\right)$ was computed. The study protocol follows the recommendations of the ethical guidelines of the 1975 Declaration of Helsinki and was approved by our ethical committee.

Histological analysis of human liver biopsies was performed by two independent pathologists. The grade of steatosis was defined as 1 when less than $30 \%$ of hepatocytes presented lipid vacuoles, and 2 when $30-60 \%$ of hepatocytes presented lipid vacuoles. Two groups of liver biopsies were considered: the first group consisted of nine liver biopsies without any histological feature of steatosis or inflammation; the second group consisted of nine liver biopsies with grade $1(n=5)$ and grade $2(n=4)$ steatosis. There was no fibrosis, Mallory bodies or cirrhosis in any of the livers used. The mean BMI was $26.5 \pm 4.3 \mathrm{~kg} / \mathrm{m}^{2}$ in the steatosis group and $22.7 \pm 3.0 \mathrm{~kg} / \mathrm{m}^{2}$ in patients without steatosis $(P<0.05)$.

A second set of liver biopsies was used for immunohistochemistry and/or quantitative RT-PCR studies. It consisted of 22 other human frozen liver biopsies from graft donors selected for the presence $(n=11)$ or absence $(n=11)$ of steatosis and the corresponding paraffin-embedded samples, and also paraffin-embedded liver samples with steatohepatitis from obese patients $(n=4)$, from alcoholic patients $(n=4)$, or HCV-associated steatosis $(n=4)$.

\section{RNA Extraction and Microarray Hybridization}

Gene expression in fatty and normal livers was analysed using a high-density oligonucleotide microarray (HG-U133A; Affymetrix, Wooburn 
Green, UK) containing 22283 sequences. Targets were prepared and microarrays were processed as described in the Affymetrix GeneChip Expression Analysis manual. Briefly, the total RNA was purified with an RNeasy kit (Qiagen, Courtaboeuf, France). In all, $5 \mu \mathrm{g}(100 \mathrm{ng} / \mu \mathrm{l})$ of total RNA were used to synthesize double-stranded cDNA with SuperScript II reverse transcriptase (Invitrogen, Cergy Pontoise, France) and a T7-(dT)24 primer (Proligo Biochemie GmbH, Hamburg, Germany). Biotinylated cRNA was synthesized from the double-stranded cDNA using the RNA Transcript Labeling kit (Enzo Life Sciences, Farmingdale, NY, USA). The biotinylated cRNA was purified and fragmented before being hybridized to the oligonucleotide microarray, which was washed and stained with streptavidin-phycoerythrin. Arrays were scanned with a GeneArray ${ }^{\mathbb{R}}$ Scanner Update apparatus (Affymetrix, Wooburn Green, UK).

\section{Analysis of Microarray Data}

Starting from the $18 \mathrm{CEL}$ files, gene expression measures were computed using the Robust Multichip Average (RMA) method described in Irizarry et al. ${ }^{14}$ This method includes the following successive steps: (1) background correction; (2) probe-level quantile normalization; ${ }^{15}$ (3) calculation of expression measures using median polish.

The first step of data analysis consisted in unsupervised hierarchical clustering of liver samples, based on gene expression measures, with no gene preselection. An agglomerative procedure (ie, a bottom-up procedure, where one starts with each sample in its own cluster and, at each step, joins the two closest clusters, until all samples are in a single cluster) was applied. The Euclidean distance was used as a dissimilarity measure between individual samples and average linkage as a dissimilarity measure between two clusters (ie, average of all distances between samples in the two clusters).

The second step of analysis consisted in the identification of differently expressed sequences between the two groups of liver samples (normal and steatotic livers). Two-sample t-statistics, with unequal variance, were computed for each sequence. The step-down maxT multiple testing procedure was used for a Family-Wise Error Rate (ie, the chance of at least one false positive) of 5\% (Type I error). ${ }^{16-18}$ This procedure takes into account the joint distribution of the test statistics and uses resampling (eg bootstrap or permutation) to estimate this distribution.

Data analysis was performed with the open-source software R, Version 1.9.0. (CRAN, www.cran. r-project.org). The function hclust from the stats package was used for hierarchical clustering. The following Bioconductor Project packages (www. bioconductor.org ${ }^{19}$ ) were used: $a f f y^{20}$ for data preprocessing, multtest ${ }^{21}$ for the identification of differentially expressed sequences and annaffy for gene annotation.

\section{Quantitative RT-PCR}

Total mRNA of genes involved in inflammation pathway (SIGIRR for single immunoglobulin (Ig) interleukin 1 receptor (IL1-R)-related molecule, TOLLIP for toll interacting protein and SITPEC for likely ortholog of mouse signalling intermediate in Toll pathway-evolutionarily conserved) were analysed using TaqMan ${ }^{\circledR}$ procedure. First-strand cDNA was synthesized with $50 \mathrm{ng}$ of RNA from liver sample in a volume of $50 \mu \mathrm{l}$ with TaqMan ${ }^{\circledR}$ reverse transcription reagents (Applied Biosystems, Foster City, USA). Reactions in which the enzyme was omitted were used as negative controls. For the standard curve, reverse transcription was performed in the same conditions with $500 \mathrm{ng}$ of RNA from healthy liver. For each series of samples, a new standard curve was prepared. Primers were chosen with the assistance of the computer program Primer Express (Applied Biosystems, Foster City, USA) Table 2. The specificity of the nucleotide sequences was confirmed by conducting BLAST searches. The 18S genomic control kit (Eurogentec, Angers, France), designed for ribosomal $18 S$ RNA amplification, was used as a reference to normalize the results. The $18 S$ cDNA PCR mixture contained $12.5 \mu \mathrm{l}$ of Mastermix Plus ${ }^{\circledR}$ reagents (Eurogentec, Angers, France), $5 \mathrm{nmol}$ of forward primer, $5 \mathrm{nmol}$ of reverse primer and $2.5 \mathrm{nmol}$ of probe to $18 \mathrm{~S}$ gene in a total volume of $25 \mu \mathrm{l}$. To quantify genes implicated in inflammation, a PCR mixture contained $2.5 \mu \mathrm{l}$ volume of reverse transcription product, $12.5 \mu \mathrm{l}$ of qPCR Mastermix Plus ${ }^{\circledR}$ for Sybergreen I without UNG (Eurogentec, Angers, France), $1 \mu \mathrm{l}$ of each primers (forward and reverse at $10 \mathrm{nmol} / \mu \mathrm{l}$ ) and $8 \mu \mathrm{l}$ of water in a total volume of $25 \mu \mathrm{l}$. For standard curve, a $2.5 \mu \mathrm{l}$ volume of pure and diluted cDNA from liver RNAs control $(1 / 10,1 / 100,1 / 1000)$ was used. Each of the 50 PCR cycles consisted of $15 \mathrm{~s}$ denaturation at $95^{\circ} \mathrm{C}$ and hybridization of probes and primers as well as DNA synthesis for $1 \mathrm{~min}$ at $60^{\circ} \mathrm{C}$. Experiments were performed in triplicate for each standard and sample data point. The relative amounts of cDNA for each gene and $18 S$ gene in the samples were computed by comparison with standard curves. For each sample, results were normalized, using the $18 S$ RNA value of the calibrator to obtain a final R-gene value.

\section{MtDNA Quantification}

We quantified mitochondrial DNA (mtDNA) in the total DNA extracted from each liver biopsy with $(n=20)$ and without $(n=20)$ steatosis using the method described before. ${ }^{22}$ In brief, the nuclear gene (TaqMan $^{\circledR} \beta$-actin control reagent, Perkin Elmer, Courtaboeuf, France) and the mitochondrial gene 
Table 2 The different primers used for quantitative RT-PCR and quantitative PCR

\begin{tabular}{|c|c|c|}
\hline Primers & Sequences of primers & Gene identification \\
\hline $\mathrm{mtDNA}^{\mathrm{a}}$ & $\begin{array}{l}\text { Sense: 5'-Mito-CytB-F-CAACATCTCCGCATGATGAAA-3' } \\
\text { Antisense: 5'- Mito-CytB-R-CCATAATTTACGTCTCGAGTGATGTG-3' } \\
\text { Probe: 5'-6-Fam-CCATGCACTACTC ACCAGACGCCTCAA-3'-Tamra }\end{array}$ & $\begin{array}{l}\text { Mitochondrial DNA } \\
\text { (cytochrome } b \text { ) } \\
\text { MT-CYB }\end{array}$ \\
\hline $\begin{array}{l}\text { Single Ig Il-1R-related } \\
\text { molecule }\end{array}$ & $\begin{array}{l}\text { Sense: } 5^{\prime} \text {-CCAGGTCCTGGCAGCAG-3' } \\
\text { Antisense: 5'-AGACTGATCCAAGAGCCTGGG-3' }\end{array}$ & SIGIRR \\
\hline TOLLIP & $\begin{array}{l}\text { Sense: 5'-AGTACGGAGGCGCAGTGG-3' } \\
\text { Antisense: } 5^{\prime} \text {-CGGGTCATGCCGTAATCTT-3' }\end{array}$ & TOLLIP \\
\hline SITPEC & $\begin{array}{l}\text { Sense: } 5^{\prime} \text {-AAGACAGAGCTGGCCTGGAGT-3' } \\
\text { Antisense: } 5^{\prime} \text {-AGTAGGGTGGCCTGGACCC-3' }\end{array}$ & SITPEC \\
\hline
\end{tabular}

SIGIRR, single Ig IL1-R-related molecule; SITPEC, likely ortholog of mouse signalling intermediate in Toll pathway-evolutionarily conserved; TOLLIP, Toll interacting protein. ${ }^{22}$

${ }^{\mathrm{a}}$ Chiappini et $a .^{22}$

MT-CYB (cytochrome $b$ or cyt $b$ ) were quantified separately by real-time quantitative PCR. We amplified part of the cyt $b$ mitochondrial gene with specific primers (Table 2). The PCR mixtures contained $7 \mu \mathrm{l}$ of water, $12.5 \mu \mathrm{l}$ of qPCR Mastermix Plus ${ }^{\circledR}$ reagents (Eurogentec, Angers, France), 0.4 $\mathrm{pmol} / \mu \mathrm{l}$ of each primer and $0.2 \mathrm{pmol} / \mu \mathrm{l}$ of each probe. Each sample was subjected to real-time PCR in triplicate. The amplification conditions are the same as described before. Fluorescence was measured at the end of each annealing step. A standard curve of 10, 100, 1000, 10000 and 100000 nucleargenome equivalents was included in each run, and the same nuclear-genome equivalent values were used to quantify both the $\beta$-actin and cyt $b$ genes. The data were expressed as the ratio of the mean mitochondrial DNA value (cyt $b$ ) of the triplicate measurements to the mean nuclear DNA value ( $\beta$-actin) of the triplicate measurements for a given extract (mtDNA/ $\beta$-actin).

\section{Immunohistochemistry}

A total of 34 formalin-fixed paraffin-embedded hepatic liver tissues identified using a computerized database were retrieved from our surgical pathology files to perform immunohistochemistry analyses. There were 11 liver samples with steatosis, 11 liver samples without steatosis, four HCV-infected livers exhibiting steatosis and eight steatohepatitis samples due to excessive alcohol consumption $(n=4)$ or obesity $(n=4)$. The tissue microarray construction was made as described by Kononen et al. ${ }^{23}$ Each case comprised a triplet of liver tissue as spots of $0.6 \mathrm{~mm}$ in diameter. Immunostaining was performed using the standard avidin-biotin peroxidase technique with antigen retrieval in accordance with the studied antibody. The primary antibodies used were an anti-mitochondrial antigen monoclonal antibody (dilution 1:50, clone 113-1; Biogenex, San Ramon,
CA, USA), an anti-TGFB1 (sc-82) polyclonal antibodies (dilution 1:100, Santa Cruz, California, CA, USA), an anti-IL1-R1 (dilution 1:400; AB-269-NA, R \& D System, Lille, France) and an anti-IGF2 (dilution 1:800; AF-292-NA, R\&D System, Lille, France). Diaminobenzidin was used as the chromogen and haematoxylin as the nuclear counterstain. For negative controls, the primary antibody was either omitted or replaced by a suitable concentration of normal IgG of the same species. The analysis was both descriptive and semiquantitative, taking into account the cellular location of the signal in addition to the percentage of positive cells scored as 0 (no staining), 1 (less than $25 \%$ of cells), 2 (between 25 and $75 \%$ ) and 3 (more than $75 \%$ ). The intensity of immunostaining was also graded 1 (weak), 2 (medium) and 3 (strong). The analysis of the three cores per samples was similar and showed a uniform degree of staining between all cores for all antibodies except for the anti-mitochondrial antigen monoclonal antibody; an average score was determined. All sections were scored independently by two pathologists.

\section{Statistical Analysis}

The mean and standard deviation were computed in group without fatty liver and group of fatty liver and the results were compared with a Student's $t$-test $(\alpha=0.05)$. The results of immunochemistry were compared with a $\chi^{2}$ test $(\alpha=0.05)$.

\section{Results}

\section{Gene Expression}

We selected liver biopsies according to the presence or absence of liver steatosis and the lack of microscopic signs of inflammation. None of the samples presented signs of fibrosis, Mallory bodies 


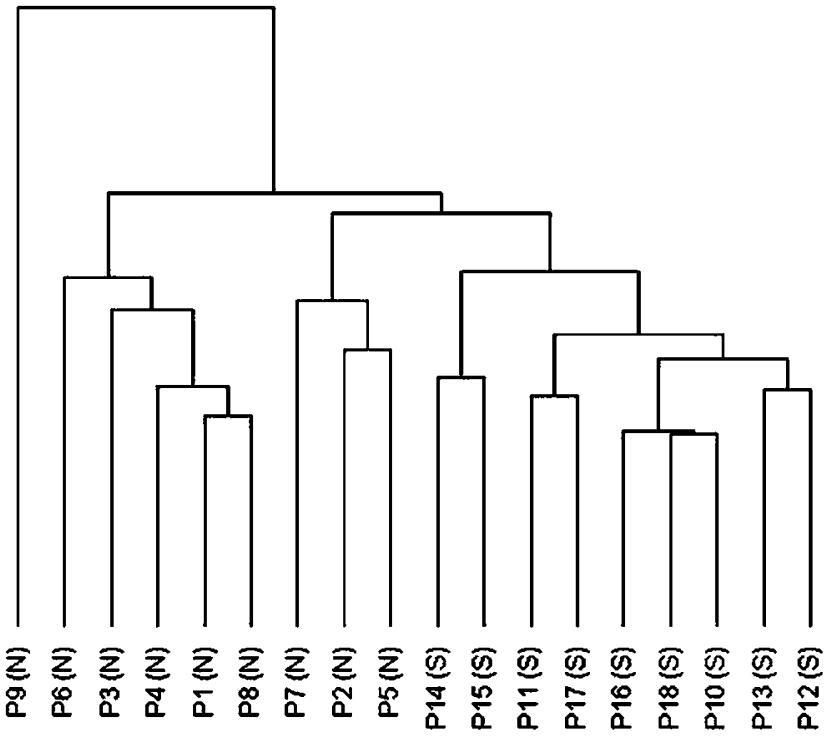

Figure 1 Hierarchical clustering of normal and steatotic liver samples. Agglomerative hierarchical clustering was performed with no gene preselection, using the Euclidean distance as a dissimilarity measure between samples, and average linkage as a dissimilarity measure between two clusters. ' $N$ ' means normal liver sample and 'S' means steatotic liver sample.

or cirrhosis. RNA was obtained from 18 human livers biopsies (nine with and nine without liver steatosis); the two groups were not different except that the BMI was significantly higher for the group of patients with liver steatosis.

RNA of each liver biopsy was hybridized to Affymetrix HG-U133A GeneChips containing approximately 22300 human transcripts. The dendrogram, which was obtained by agglomerative hierarchical clustering of liver samples with no gene selection, is shown in Figure 1. The nine steatotic liver samples clustered together, while the nine normal liver samples seemed to have more heterogeneous gene expression profiles.

According to the step-down maxT multiple testing procedures for control of the Family-Wise Error Rate (with a Family-Wise Error Rate of 5\%, ie, a 5\% probability of having at least one Type I error), 110 sequences were found to be differentially expressed in normal and steatotic liver samples. Of these, 100 sequences were overexpressed and 10 were underexpressed in steatotic liver samples. Lists of overand underexpressed sequences are given in Table 3. Figure 2 shows the expression profiles of these 110 sequences in the 18 liver samples.

Genes were classified in nine families according to their function (Table 3). One of these families included genes coding for the mitochondria respiratory chain proteins and enzymes involved in the metabolism of lipids and creatine synthesis. Another family included genes coding for ions transporters, lipids transporters (apolipoprotein $C-I V$ involved in formation of very low density lipoprotein, whereas apolipoprotein $E$ and scavenger

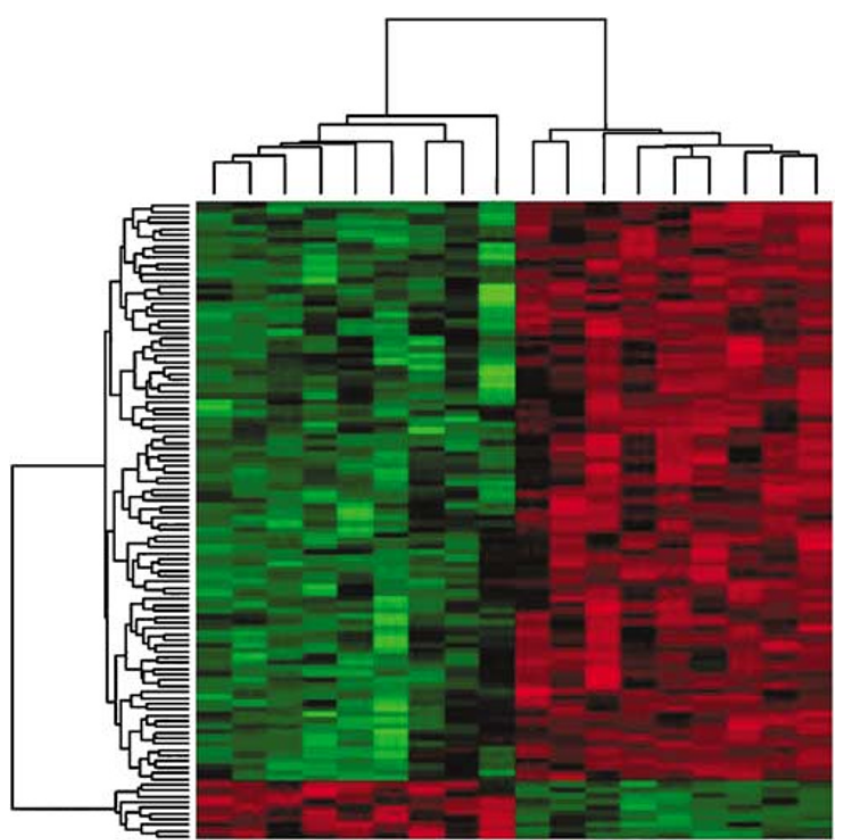

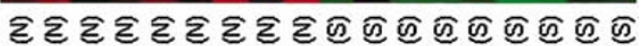

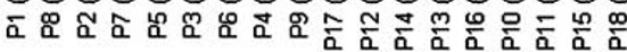

Figure 2 Expression profiles of the 110 differentially expressed sequences $(F W E R=0.05)$ in the nine normal and nine steatotic liver samples. Each row represents a probe sequence and each column represents a liver sample. The expression measures are standardized for each probe sequence (ie, row standardized). A green to red colour palette is used to represent expression levels, with green representing underexpression and red representing overexpression. Gene names are listed in Table 2. ' $\mathrm{N}$ ' means normal liver sample and ' $\mathrm{S}$ ' means steatotic liver sample.

receptor $B$ type 1 involved in selective cholesterol uptake). A majority of differentially expressed genes belonged to the families of transcription factors (nuclear and mitochondrial transcription factors) or coded for proteins involved in the cell cycle control (signal transduction, apoptosis and cell cycle), protein modifications and extracellular matrix remodelling. Two groups of genes were involved in either DNA damage repair pathways or inflammatory pathways; these are TOLLIP, single Ig IL-1R-related molecule (SIGIRR) and TGFB1.

\section{Mitochondria and Mitochondrial DNA Content in Normal and Fatty Livers}

Quantitative real-time PCR was used to determine the mtDNA to DNA ratio in 40 liver biopsies, including the 18 biopsies of the gene profiling study and 22 additional samples. The mean ratios of mtDNA to DNA content were $0.67 \pm 0.10$ and $1.12 \pm 0.14$ in the group of liver biopsies without and with steatosis, respectively $(P=0.011$, Table 4$)$. This result was supported by mitochondria immunostaining using tissue microarray analysis (Figure 3). Indeed, an increase of the number of mitochondria in hepatocytes with fatty droplet (macrovesicular 
Table 3 Differentially expressed sequences

Overexpressed sequences in steatotic liver samples

\begin{tabular}{|c|c|c|}
\hline GenBank ID & $\begin{array}{l}\text { Adjusted } \\
\mathrm{P} \text {-value }\end{array}$ & Gene description \\
\hline \multicolumn{3}{|l|}{ Metabolism } \\
\hline NM_000755 & 0.0389 & Carnitine acetyltransferase \\
\hline BC000723 & 0.0261 & Carnitine acetyltransferase \\
\hline NM_000156 & 0.0108 & Guanidinoacetate $N$-methyltransferase \\
\hline NM_003748 & 0.001 & Aldehyde dehydrogenase 4 family, member A1 \\
\hline AF092131 & 0.0177 & NADH dehydrogenase (ubiquinone) flavoprotein $1,51 \mathrm{kDa}$ \\
\hline NM_004146 & 8.00E-04 & NADH dehydrogenase (ubiquinone) 1 beta subcomplex, 7, $18 \mathrm{kDa}$ \\
\hline NM_003365 & 0.0095 & Ubiquinol-cytochrome $c$ reductase core protein I \\
\hline D83782 & 0.0194 & SREBP cleavage-activating protein \\
\hline AB016531 & 0.0132 & Peroxisomal biogenesis factor 16 \\
\hline AA523441 & 0.0118 & Peroxisomal biogenesis factor 16 \\
\hline \multicolumn{3}{|l|}{ Transport } \\
\hline AF112472 & 0.0075 & Calcium/calmodulin-dependent protein kinase (CaM kinase) II beta \\
\hline NM_003227 & 0.0237 & Transferrin receptor 2 \\
\hline NM_004925 & 0.0291 & Aquaporin 3 \\
\hline BC065834 & 0.0048 & Glutamate receptor, ionotropic, $N$-methyl D-asparate-associated protein 1 (glutamate binding) \\
\hline U79458 & 0.0016 & WW domain-binding protein 2 \\
\hline NM_024874 & 0.0029 & Polycystic kidney disease 1-like \\
\hline D87920 & 0.0448 & Solute carrier family 5 (sodium iodide symporter), member 5 \\
\hline AI358867 & 0.077 & Apolipoprotein C-IV \\
\hline NM_000041 & 0.0381 & Apolipoprotein E \\
\hline NM_005505 & $3.00 \mathrm{E}-04$ & Scavenger receptor class $\mathrm{B}$, member 1 \\
\hline AF009670 & 0.0158 & ATP-binding cassette, subfamily C (CFTR/MRP), member 3 \\
\hline NM_000263 & 0.0075 & $N$-acetylglucosaminidase, alpha- (Sanfilippo disease IIIB) \\
\hline NM_021126 & 0.0203 & Mercaptopyruvate sulphurtransferase \\
\hline \multicolumn{3}{|c|}{ Mitochondrial transcription regulation } \\
\hline NM_015950 & 0.0037 & Mitochondrial ribosomal protein L2 \\
\hline NM_002949 & 0.0076 & Mitochondrial ribosomal protein L12 \\
\hline AI832239 & 0.0307 & Mitochondrial ribosomal protein L23 \\
\hline \multicolumn{3}{|c|}{ Nuclear transcription regulation } \\
\hline AL042496 & 0.0132 & Chromosome 9 open reading frame 7 \\
\hline NM_017586 & 0.0018 & Chromosome 9 open reading frame 7 \\
\hline BC000744 & 0.0242 & Ubiquitin-conjugating enzyme E2I (UBC9 homolog, yeast) \\
\hline NM_015925 & 0.0455 & Liver-specific bHLH-Zip transcription factor \\
\hline NM_016011 & 0.0029 & Nuclear receptor-binding factor 1 \\
\hline NM_005439 & 0.0242 & Myeloid leukaemia factor 2 \\
\hline AL096741 & 0.0206 & ASC-1 complex subunit P100 \\
\hline AF104913 & 0.0022 & Eucaryotic translation initiation factor 4 gamma, 1 \\
\hline NM_005851 & 0.0178 & Tumour suppressor deleted in oral cancer-related 1 \\
\hline BC002356 & 0.0035 & Nucleobindin 1 \\
\hline AL046054 & $1.00 \mathrm{E}-04$ & Prostate tumour overexpressed gene 1 \\
\hline NM_001662 & 0.0132 & ADP-ribosylation factor 5 \\
\hline U56418 & 0.0014 & 1-acylglycerol-3-phosphate $O$-acyltransferase 2 (lysophosphatidic acid acyltransferase, beta) \\
\hline
\end{tabular}

Signal transduction/apoptosis/cell cycle

$\begin{array}{lll}\text { AF202063 } & 0.0022 & \text { Fibroblast growth factor receptor 4 } \\ \text { BF940276 } & 0.0086 & \text { Radical fringe homologue (Drosophila) } \\ \text { AU159484 } & 0.0123 & \text { Glucocorticoid receptor DNA-binding factor 1 } \\ \text { U03469 } & 0.0422 & \text { Glucagon receptor } \\ \text { X07868 } & 9.00 E-04 & \text { Insulin-like growth factor 2 (somatomedin A) } \\ \text { AW338791 } & 0.0041 & \text { Insulin-like growth factor-binding protein, acid } \\ \text { M17863 } & 0.0181 & \text { Insulin-like growth factor 2 (somatomedin A) } \\ \text { NM_001552 } & 0.0468 & \text { Insulin-like growth factor-binding protein 4 } \\ \text { NM_001654 } & 0.0345 & \text { v-raf murine sarcoma 3611 viral oncogene homo } \\ \text { NM_005163 } & 0.0255 & \text { v-akt murine thymoma viral oncogene homologu } \\ \text { AF035625 } & 0.0186 & \text { Serine/threonine kinase 11 (Peutz-Jeghers syndrongending protein } \\ \text { AI689402 } & 0.0169 & \text { Putative NF } \kappa \text { B-activating proteing alpha } \\ \text { BF570122 } & 0.0166 & \text { Peptidase (mitochondrial processing } \\ \text { AF274972 } & 0.0317 & \text { Leucine-rich and death domain containing } \\ \text { BG476661 } & 0.0406 & \text { Cell division cycle 34 } \\ \text { NM_007061 } & 0.0038 & \text { CDC42 effector protein (Rho GTPase binding) 1 } \\ \text { AF153430 } & 8.00 E-04 & \text { Cyclin-dependent kinase (CDC2-like) 10 } \\ \text { NM_022372 } & 0.0315 & \text { G protein beta subunit-like }\end{array}$


Table 3 Continued

Overexpressed sequences in steatotic liver samples

\begin{tabular}{|c|c|c|}
\hline GenBank ID & $\begin{array}{l}\text { Adjusted } \\
\text { P-value }\end{array}$ & Gene description \\
\hline \multicolumn{3}{|c|}{ DNA damaging repair pathway } \\
\hline AI673553 & 0.0494 & Mediator of DNA damage checkpoint 1 \\
\hline U38980 & 0.0039 & Postmeiotic segregation increased 2-like 11 \\
\hline NM_006230 & 0.0494 & Polymerase (DNA directed), delta 2, regulatory subunit $50 \mathrm{kDa}$ \\
\hline NM_021173 & 0.0022 & Polymerase (DNA-directed), delta 4 \\
\hline \multicolumn{3}{|c|}{ Defense/stress/inflammation responses } \\
\hline AB019695 & $7.00 \mathrm{E}-04$ & Thioredoxin reductase 2 \\
\hline AL136616 & 0.0015 & Proline synthetase cotranscribed homologue (bacterial) (HIF) \\
\hline AI991887 & 0.0405 & 5-oxoprolinase (ATP-hydrolysing) \\
\hline NM_016581 & 0.0046 & Likely ortholog of mouse signalling intermediate in Toll pathway-evolutionarily conserved \\
\hline NM_019009 & 0.0481 & Toll interacting protein \\
\hline NM_021805 & 0.0459 & Single Ig IL-1R-related molecule \\
\hline AI560217 & 0.0405 & Interleukin 17 receptor $\mathrm{C}$ \\
\hline NM_004740 & 0.0355 & TGFB1-induced antiapoptotic factor 1 \\
\hline NM_000063 & 0.0278 & Complement component 2 \\
\hline M17263 & 0.0112 & Complement component 8, gamma polypeptide \\
\hline AB008047 & 0.0282 & Mannan-binding lectin serine protease 2 \\
\hline \multicolumn{3}{|c|}{ Cellular adhesion/cytoskeleton } \\
\hline NM_022834 & 0.0027 & Hypothetical protein FLJ22215 \\
\hline NM_004475 & $1.00 \mathrm{E}-04$ & Flotillin 2 \\
\hline NM_002428 & 0.0023 & Matrix metalloproteinase 15 (membrane-inserted) \\
\hline AL031282 & 0.0234 & Matrix metalloproteinase 23B \\
\hline NM_030582 & 0.0158 & Collagen, type XVIII, alpha 1 \\
\hline NM_000918 & 0.0103 & Procollagen-proline, 2-oxoglutarate 4-dioxygenase \\
\hline J02783 & 0.0017 & Procollagen-proline, 2-oxoglutarate 4-dioxygenase \\
\hline AV693216 & 0.0103 & Plexin B1 \\
\hline NM_002151 & 0.0246 & Hepsin (transmembrane protease, serine 1) \\
\hline NM_001306 & 0.0087 & Claudin 3 \\
\hline NM_002997 & 0.0397 & Syndecan 1 \\
\hline BC001165 & 0.0019 & N-ethylmaleimide-sensitive factor attachment protein, alpha \\
\hline AI762627 & 0.0184 & Protein tyrosine phosphatase, receptor type, F \\
\hline \multicolumn{3}{|c|}{ Protein synthesis and modification } \\
\hline AW584011 & 7.00E-03 & Similar to chymotrypsinogen B precursor \\
\hline D86062 & 0.0313 & Chromosome 21 open reading frame 33 \\
\hline NM_017670 & 0.0018 & Ubiquitin-specific protease otubain 1 \\
\hline AF059752 & 0.0428 & Mannose-P-dolichol utilization defect 1 \\
\hline \multicolumn{3}{|l|}{ Unknown } \\
\hline NM_024648 & 0.0109 & Hypothetical protein FLJ22222 \\
\hline NM_024710 & $2.00 \mathrm{E}-04$ & Hypothetical protein FLJ23469 \\
\hline NM_024527 & 0.0038 & Hypothetical protein FLJ11743 \\
\hline NM_024671 & 0.026 & Hypothetical protein FLJ23436 \\
\hline BF055496 & 0.0023 & DKFZP586J0619 protein \\
\hline BG255188 & 0.0056 & NA \\
\hline NM_013265 & 0.0302 & Chromosome 11 open reading frame 2 \\
\hline $\mathrm{Z} 78349$ & 0.0484 & Hypothetical protein MGC3262 \\
\hline NM_023933 & 0.0102 & Hypothetical protein MGC2494 \\
\hline ВC004409 & 0.0076 & Hypothetical protein BC004409 \\
\hline AL136683 & 0.0079 & Hypothetical protein DKFZp564D0478 \\
\hline
\end{tabular}

$\begin{array}{lll}\text { Signal transduction/apoptosis/cell cycle } & \\ \text { NM_001166 } & 0.0047 & \text { Baculoviral IAP repeat-containing } 2 \\ \text { NM_020239 } & 0.0228 & \text { Small protein effector 1 of Cdc42 } \\ \text { BE737620 } & 0.0194 & \text { Protein phosphatase 1, regulatory (inhibitor) subunit 12A }\end{array}$

Nuclear transcription regulation BE737027 $\quad 0.0309$ NM $005087 \quad 9,00 \mathrm{E}-04$ NM_002266 $\quad 0.0049$ U83115 0.0399
Mediator of DNA damage checkpoint 1

regulatory subunit $50 \mathrm{kDa}$

Thioredoxin reductase 2

roline synthetase cotranscribed homologue (bacterial) (HIF

Single Ig IL-1R-related molecule

Complement component 2

Complement component 8 , gamma polypeptide

Hypothetical protein FLJ22215

Flotillin 2

Matrix metalloproteinase 23B

Procollagen-proline, 2-oxoglutarate 4-dioxygenase

Procollagen-proline, 2-oxoglutarate 4-dioxygenase

Claudin 3

Syndecan 1

N-ethylmaleimide-sensitive factor attachment protein, alpha

Similar to chymotrypsinogen B precursor

open reading frame 33

Hypothetical protein FLJ22222

DKFP586J0619 protein

Hypothetical protein MGC3262

Underexpressed sequences in steatotic liver samples 
Table 3 Continued

Underexpressed sequences in steatotic liver samples

\begin{tabular}{lll}
\hline GenBank ID & $\begin{array}{l}\text { Adjusted } \\
\text { P-value }\end{array}$ & Gene description \\
& \\
\multicolumn{2}{l}{ Cellular adhesion/cytoskeleton } \\
AL110164 & 0.0049 & LIM and senescent cell antigen-like domains 1 \\
& & \\
Transporter & & Chloride intracellular channel 4 \\
NM_013943 & 0.0057 & Solute carrier family 39 (metal ion transporter), member 6 \\
AI635449 & 0.0025 &
\end{tabular}

Table 4 Quantification of genes involved in inflammation pathway (SITPEC, SIGIRR, TOLLIP) and the content of mtDNA in livers with and without steatosis

\begin{tabular}{lccc}
\hline & \multicolumn{3}{c}{ Patients } \\
\cline { 2 - 4 } & $\begin{array}{c}\text { Without steatosis } \\
(\mathrm{N}=20)\end{array}$ & $\begin{array}{c}\text { With steatosis } \\
(\mathrm{N}=20)\end{array}$ & $\mathrm{P}$ \\
\hline SITPEC & $0.90 \pm 0.19$ & $1.71 \pm 1.03$ & 0.05 \\
SIGIRR & $0.48 \pm 0.24$ & $0.74 \pm 0.30$ & 0.05 \\
TOLLIP & $1.41 \pm 0.54$ & $3.28 \pm 2.24$ & 0.05 \\
Ratio mtDNA/ & $0.67 \pm 0.10$ & $1.12 \pm 0.14$ & 0.01 \\
$\beta$-actin & & & \\
\hline
\end{tabular}

steatosis) was observed using deep stain and the counting of the number of brown points (Figure 3c and d). In normal liver, the stain was spread within the liver tissue (Figure 3a and b). A semiquantitative evaluation of the staining showed a significant difference between the two groups (two positive samples of the 11 normal livers vs nine positive of the 11 fatty livers; $\chi^{2} ; P<0.01$ )

\section{Validation of the Expression of Steatosis-Related Genes in Additional Liver Samples}

Using TaqMan ${ }^{\circledR}$ procedure, we quantified three genes involved in the inflammation response that exhibited a highly significant differential expression in normal and fatty livers (Table 2). There were likely ortholog of mouse signalling intermediate in Toll pathway-evolutionarily conserved (SITPEC), toll interacting protein (TOLLIP) and SIGIRR mRNA. We confirmed an increase in the mRNA expression of these genes in fatty liver compared to normal livers $(1.71 \pm 1.03$ vs $0.90 \pm 0.19 ; \quad 3.28 \pm 2.24 \quad$ vs $1.41 \pm 0.54 ; 0.74 \pm 0.30$ vs $0.48 \pm 0.24$, respectively, Table 4). We have analysed the association between the increased expression of these genes and the BMI. We have only observed a trend for a correlation between TOLLIP mRNA levels and BMI $(P=0.055)$. Of interest was the increase of the expression of mitochondrial antigen, IL-1R1, IGF2 and TGFB1 in most of the HCV-associated steatosis and steato- hepatitis. IL1-R1 was always strongly expressed in steatohepatitis linked to alcohol or obesity and in half of the HCV-associated steatosis (Table 5). Figure 4 shows a uniform spread of IL1-R1 staining in fatty liver tissue. In contrast, IGF2 was expressed in normal livers and only an increase in the immunostaining intensity was observed.

\section{Discussion}

The current understanding of the mechanisms by which triglycerides accumulate within the liver and by which hepatic steatosis progresses to steatohepatitis is based almost exclusively on data from animal models. Liver biopsies are rarely performed in patients with fatty livers. Thus, we have performed genome expression profiling studies in patients to better understand the molecular basis of liver steatosis per se. We studied human liver biopsies with simple fat deposition excluding steatosis with microscopic signs of inflammation. All of the patients included in the study had stayed for less than $24 \mathrm{~h}$ in the intensive care unit, to exclude patients with long fasting or intravenous glucose perfusion to avoid the depletion in liver glycogen content. However, the statistical analysis of the differentially expressed genes between the two groups of patients who stayed for similar durations in intensive care and who have received similar medications avoids the artefact of differential expression of genes linked to starvation due to the intensive care stay. Moreover, the dendrogram of the liver samples, by agglomerative hierarchical clustering, including all 22300 sequences, validates our histological selection of them. Indeed, it shows that all of the steatosis liver samples clustered together, compared to liver without steatosis. It appears that this homogenous classification of steatosis biopsies may originate from an identical metabolic disruption. Our population of donors of liver grafts preferentially exhibited steatosis linked to excess body fat since their BMIs were significantly higher than those of patients without liver steatosis. Some of them may have had high alcohol consumption. However, none of the patients were infected with HBV, HCV or HIV. Thus, our population can be 

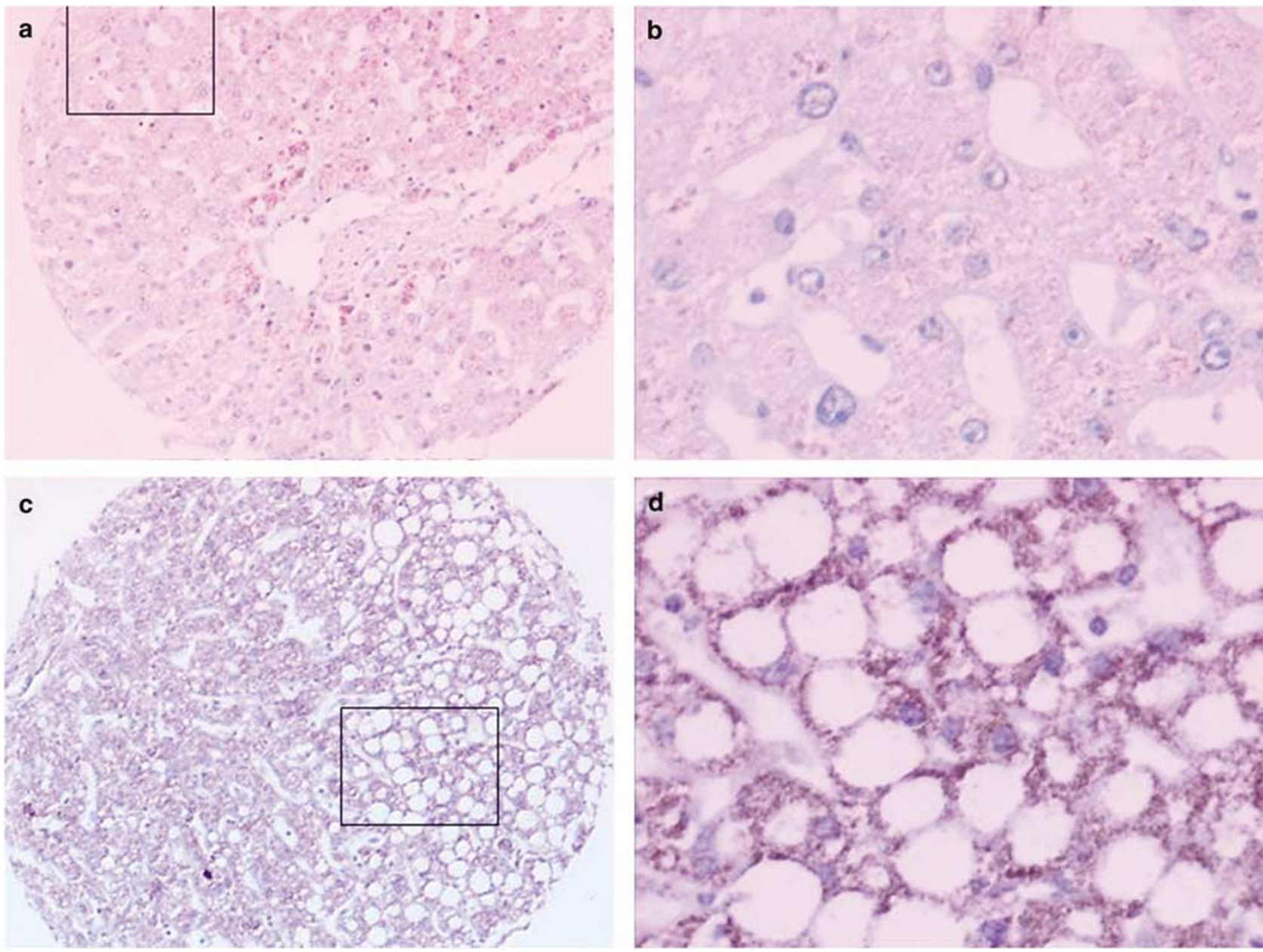

Figure 3 Examples of mitochondria immunostaining (brown) by specific anti-mitochondria antibody in normal liver (a, b) and in macrovesicular steatosis liver (c, d) from tissue microarray. Nucleus was stained by haematoxylin (blue). (a) Immunostaining is spread in normal liver tissue $(\times 20)$ going away from portal vein. $(\mathbf{b})(\times 40)$ represents an enlargement of the square from (a) and shows a stain spread in hepatocytes. (c) Immunostaining is focused in steatosis liver tissue $(\times 20)$. $(\mathbf{d})(\times 40)$ represents an enlargement of the square from (c) and shows a stain focused around the nucleus and vacuole with strong stain intensity.

Table 5 Pattern of expression of four proteins assessed by immunohistochemistry on tissue microarray as described in Materials and methods

\begin{tabular}{|c|c|c|c|c|c|c|c|c|c|c|c|c|}
\hline \multirow[t]{2}{*}{ Positive immunostaining } & \multicolumn{3}{|c|}{ Mitochondrial antigen } & \multicolumn{3}{|c|}{ IL1-R1 } & \multicolumn{3}{|c|}{ TGFB1 } & \multicolumn{3}{|c|}{$I G F-I I$} \\
\hline & $\mathrm{n}$ & $\%$ & Intensity & $\mathrm{n}$ & $\%$ & Intensity & $\mathrm{n}$ & $\%$ & Intensity & $n$ & $\%$ & Intensity \\
\hline \multicolumn{12}{|l|}{ Control } & $1-2$ \\
\hline Steatosis & & & & & & & & & & & & \\
\hline $\begin{array}{l}n=11 \\
\text { HCV-related steatosis }\end{array}$ & 9 & 82 & $1-3$ & 11 & 100 & $2-3$ & 11 & 100 & $2-3$ & 11 & 100 & $1-3$ \\
\hline $\begin{array}{l}n=4 \\
\text { ASH }\end{array}$ & 3 & 75 & $2-3$ & 2 & 50 & $2-3$ & 4 & 100 & $2-3$ & 4 & 100 & 3 \\
\hline $\begin{array}{l}\quad n=4 \\
\text { NASH }\end{array}$ & 4 & 100 & $2-3$ & 4 & 100 & $2-3$ & 3 & 75 & $2-3$ & 4 & 100 & 3 \\
\hline$n=4$ & 3 & 75 & $2-3$ & 4 & 100 & 3 & 3 & 75 & $2-3$ & 4 & 100 & 3 \\
\hline
\end{tabular}

ASH, alcoholic steatohepatitis; NASH, nonalcoholic steatohepatitis associated with obesity.

considered a general population with little risk for development of NASH. Indeed, none of the biopsies exhibited histological features of inflammation.
In 1998, a 'two-hit' model was proposed to explain the pathogenesis of steatohepatitis, the first hit being steatosis, which increases the sensitivity of 

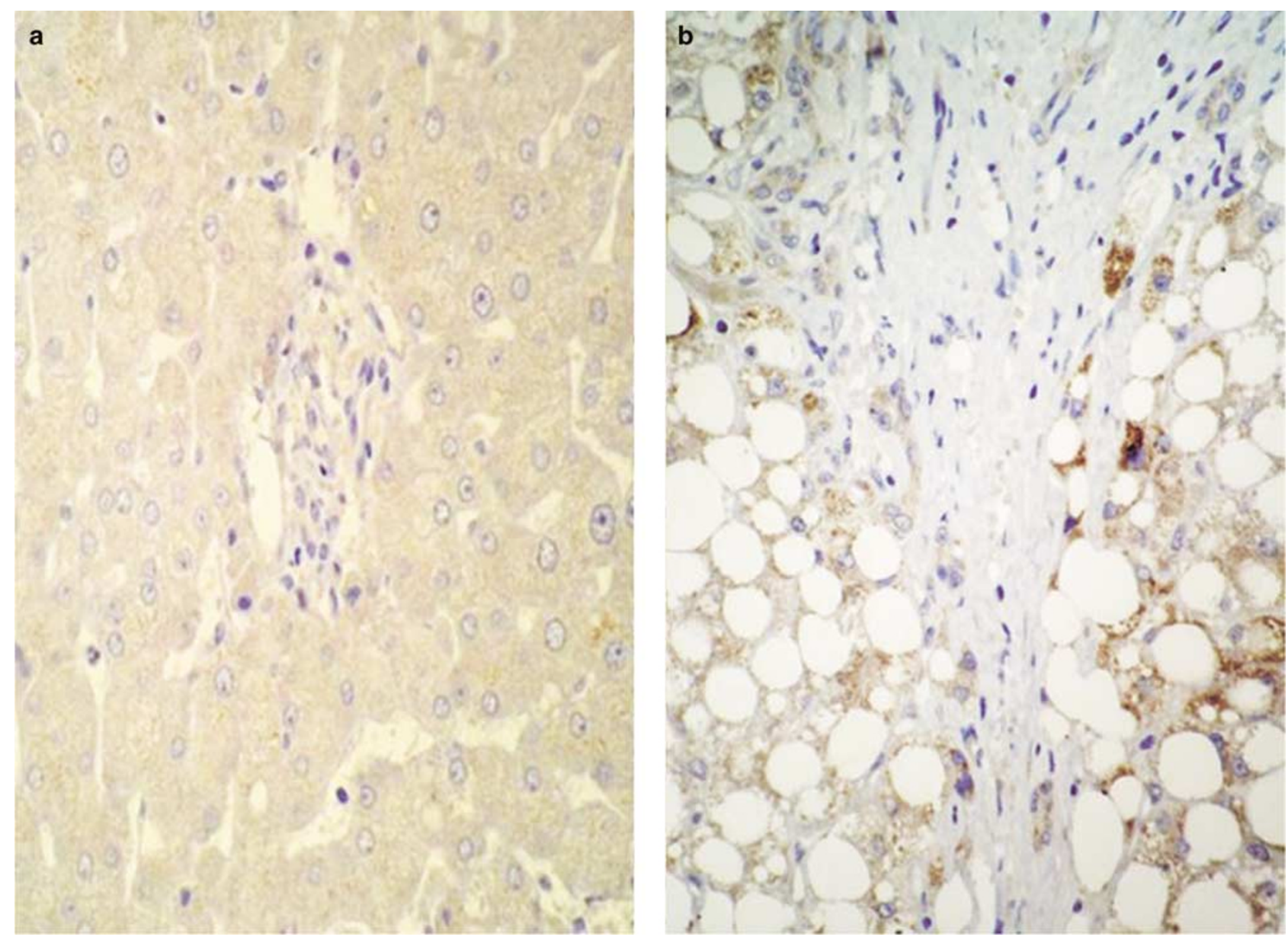

Figure 4 IL1-R1 is expressed in the cytoplasm of hepatocytes in both normal (a) and fatty liver (b). Immunoreactivity is more intense in steatotic samples. (a, b) $\times 20$.

the liver to the second hit, which mediates liver injury. More recent data have emphasized the critical role of free fatty acids from adipose tissue and insulin resistance. However, these conclusions were mostly drawn by combining results obtained in animal models with the limited amount of human data available. Recently, Sreekumar et $a 1^{13}$ studied global gene expression in liver biopsies from humans with NASH and emphasized the role of impaired mitochondrial function with attenuated capacity for dismutation of reactive oxygen species and diminished insulin sensitivity. In our study, many of the 110 differentially expressed genes were involved in mitochondrial metabolism, nine of them coding for proteins involved in the mitochondrial respiratory chain and some others encoding proteins that can interfere with mitochondrial metabolism. The increased metabolic capacity in mitochondrial metabolism is corroborated by the significant increase in the number of mitochondria per fatty cell as well as in the mitochondrial DNA content to nuclear DNA ratio in liver steatosis compared to normal livers. By contrast, a few genes were involved in the response to increased reactive oxygen species generation and the subsequent lipid peroxidation due to an increased fatty acid $\beta$ oxidation and peroxisomal fatty acid oxidation, which is one of the proposed 'second hit' mechanisms, as shown in the study on gene expression in NASH. ${ }^{24,25}$ We did not observe any difference in gene expression that may further uncouple oxidation and phosphorylation and thus worsen the oxidative stress, as observed in animals. ${ }^{26}$ This may be because we selected liver biopsies without any signs of inflammation.

Steatohepatitis is characterized by the presence of fibrosis. However, little is known about the mechanisms of fibrogenesis linked to steatosis in human liver. The most frequently proposed mechanism is the activation of hepatic stellate cells by cytokines and oxidative stress ${ }^{5,7,24,25}$ as well as an increase in the amount of connective tissue growth factor $\left(\mathrm{CTGF},{ }^{27}\right)$ and leptin, ${ }^{28}$ leading to the activation of TGFB1. ${ }^{29}$ In our study, a small number of genes implicated in the inflammatory process were significantly up- or downregulated. However, TGFB1, which is an important mediator of the mesenchymal-epithelial interactions responsible for context-dependent inhibition or stimulation of cell proliferation ${ }^{29-31}$ and also neoplastic transformation, 
was strongly overexpressed in fatty livers. A corresponding increase of TGFB1 at the protein levels was observed in additional samples including steatosis and steatohepatitis, or in all the samples of steatosis linked to HCV viral infection, suggesting its role in the latter pathology. We also observed the overexpression of a few genes coding for proteins involved in intercellular adhesion, protease activity or actin cytoskeleton, for example, collagen XVIII, syndecan 1, matrix metalloproteinase $23 B$, interleukin 17 receptor $C$ and single Ig $I L-1 R$. Indeed, the Toll/Interleukin-1 receptor (TIR) family is a major cytokine receptor family including IL-1 receptor subfamily, MyD88 family and Toll-like receptor subfamily involved in the development of tissue inflammation. Our results show that TGFB1, likely ortholog of mouse signalling intermediate in Toll pathway-evolutionarily conserved (SITPEC), toll interacting protein (TOLLIP) and single Ig IL-1Rrelated molecule (SIGIRR) gene expression as confirmed both by mRNA quantification using QRTPCR and immunhistochemistry were increased in fatty livers. This overexpression could be attributed to the activation of the Kupffer cells or hepatocytes releasing interleukins that react with hepatic stellate cells as recently suggested by Tilg and Diehl (review $^{31,32}$ ). On tissue arrays, we have observed an overexpression of IL1-R1 in all the steatosis of liver graft donors as well as in all the steatohepatitis samples associated with either excessive alcohol consumption or obesity showing that the overexpression of IL-1R1 could be a major pathway activated early in the development of steatohepatitis. The detection of IL-1R1 could be an accurate and early marker in patients at risk to develop a steatohepatitis.

Our results also suggest that the extracellular matrix could be altered since the early steps of FLD. This could be due to elevated concentrations of intracellular fatty acids within the liver parenchyma that have already been shown to be directly toxic to hepatocytes, leading to oxidative stress, inflammation and fibrogenesis, but not to mitochondrial toxicity. ${ }^{33,34}$ This could also be due to the overexpression of genes such as IGF2, IGFB4 and IGFBP acid labile unit or Claudin3 or hepsin acting through TGFB1 activation of Kupffer cells/macrophages or stellate cells leading to remodelling of extracellular matrix or activating cells to growth as in liver regeneration or activating apoptosis in hepatic cells to control the hepatocyte mass. ${ }^{35-38}$ TGFB1 and IGF2 protein expression was increased in most of the samples exhibiting either steatosis or steatohepatitis and corroborate our results of gene expression profiling. However, the basal expression of IGF2 in nonsteatotic livers led to difficulties in the interpretation of the results.

In conclusion, our results reveal that mitochondria play a major role since the early steps of steatosis, perhaps to ensure the catabolism of hepatic lipids, with increased mitochondrial phos- phorylative and oxidative metabolism. Although no microscopic signs of inflammation were observed on liver biopsies, an increase in the expression of genes or proteins involved in inflammation pathways and/ or extracellular matrix remodelling, such as TGFB1, IL1-R1 and IGF2, was observed in fatty livers. IL1-R1 seems to play a role in the development of both steatosis and steatohepatitis and could be considered as an early and accurate marker in patients at risk to develop NASH.

\section{Acknowledgements}

Franck Chiappini was supported by a grant from Association pour la Recherche sur le Cancer (ARC). Alain Barrier was supported by a grant from la Ligue Contre le Cancer. Marie-Charlotte Domart was supported by a grant from Association pour la Nouvelle Recherche Biomédicale (ANRB)-Vaincre le Cancer.

\section{References}

1 Reid AE. Nonalcoholic steatohepatitis. Gastroenterology 2001;121:710-723.

2 Diehl AM. Nonalcoholic steatohepatitis. Semin Liver Dis 1999;19:221-229.

3 Flejal KM, Carroll MD, Ogden CL, et al. Prevalence and trends in obesity among US adults, 1999-2000. J Am Med Assoc 2002;288:1723-1727.

4 Bianchini F, Kaaks R, Vainio H. Overweight, obesity, and cancer risk. Lancet Oncol 2002;3:565-574.

5 Powell EE, Cooksley WG, Hanson R, et al. The natural history of nonalcoholic steatohepatitis: a follow-up study of forty-two patients for up to 21 years. Hepatology 1990;11:74-80.

6 Brunt EM, Janney CG, Di Bisceglie AM, et al. Nonalcoholic steatohepatitis: a proposal for grading and staging the histological lesions. Am J Gastroenterol 1999;94:2467-2474.

7 Matteoni CA, Younossi ZM, Gramlich T, et al. Nonalcoholic fatty liver disease: a spectrum of clinical and pathological severity. Gastroenterology 1999;116: 1413-1419.

8 Bugianesi E, Leone N, Vanni E, et al. Expanding the natural history of nonalcoholic steatohepatitis: from cryptogenic cirrhosis to hepatocellular carcinoma. Gastroenterology 2002;123:134-140.

9 Caldwell SH, Oelsner DH, Iezzoni JC, et al. Cryptogenic cirrhosis: clinical characterization and risk factors for underlying disease. Hepatology 1999;29: 664-669.

10 Poonawala A, Nair SP, Thuluvath PJ. Prevalence of obesity and diabetes in patients with cryptogenic cirrhosis: a case-control study. Hepatology 2000;32: 689-692.

11 Ong J, Younossi ZM, Reddy V, et al. Cryptogenic cirrhosis and posttransplantation nonalcoholic fatty liver disease. Liver Transplant 2001;7:797-801.

12 Bradbury MW, Berk PD. Lipid metabolism in hepatic steatosis. Clin Liver Dis 2004;8:639-671. 
13 Sreekumar R, Rosado B, Rasmussen D, et al. Hepatic gene expression in histologically progressive nonalcoholic steatohepatitis. Hepatology 2003;38:244-251.

14 Irizarry RA, Hobbs B, Collin F, et al. Exploration, normalization, and summaries of high density oligonucleotide array probe level data. Biostatistics 2003;4:249-264.

15 Bolstad BM, Irizarry RA, Astrand $\mathrm{M}$, et al. A comparison of normalization methods for high density oligonucleotide array data based on variance and bias. Bioinformatics 2003;19:185-193.

16 Westfall PH, Young SS. Resampling-Based Multiple Testing. Examples and Methods for P-Value Adjustment. John Wiley \& Sons: New York, NY, 1993.

17 Dudoit S, Van der Laan MJ, Pollard KS. Multiple testing. Part I. Single-step procedures for control of general Type I error rates. Statist Appl Genet Mol Biol 2004;3 Article 13.

18 Van der Laan MJ, Dudoit S, Pollard KS. Multiple testing. Part I. Step-down procedures for control of the Family-Wise Error Rate. Statist Appl Genet Mol Biol 2004;3 Article 14.

19 Gentleman RC, Carey VJ, Bates DM, et al. Bioconductor: open software development for computational biology and bioinformatics. Genome Biol 2004;5:R80.

20 Gautier L, Cope L, Bolstad BM, et al. Affy-analysis of Affymetrix GeneChip data at the probe level. Bioinformatics 2004;20:307-315.

21 Ge Y, Dudoit S, Speed TP. Resampling-based multiple testing for microarray data analysis (with discussion). TEST 2003;12:1-78.

22 Chiappini F, Teicher E, Saffroy R, et al. A prospective evaluation of blood concentration of mitochondrial DNA as a marker of toxicity in 157 consecutively recruited untreated or HAART-treated HIV-positive patients. Lab Invest 2004;84:908-914.

23 Kononen J, Bubendorf L, Kallioniemi A, et al. Tissue microarrays for high-throughput molecular profiling of tumor specimens. Nat Med 1998;4:844-847.

24 Day CP, James OF. Steatohepatitis: a tale of two 'hits'? Gastroenterology 1998;114:842-845.

25 Day CP. Non-alcoholic steatohepatitis (NASH): where are we now and where are we going? Gut 2002;50:585-588.
26 Edvardsson U, Bergstrom M, Alexandersson M, et al. BRL49653), a PPARgamma-selective agonist, causes peroxisome proliferator-like liver effects in obese mice. J Lipid Res 1999;40:1177-1184.

27 Paradis V, Perlemuter G, Bonvoust F, et al. High glucose and hyperinsulinemia stimulate connective tissue growth factor expression: a potential mechanism involved in progression to fibrosis in nonalcoholic steatohepatitis. Hepatology 2001;34: 738-744.

28 Leclercq IA, Farrell GC, Schriemer R, et al. Leptin is essential for the hepatic fibrogenic response to chronic liver injury. J Hepatol 2002;37:206-213.

29 Kharbanda KK, Rogers II DD, Wyatt TA, et al. Transforming growth factor-beta induces contraction of activated hepatic stellate cells. J Hepatol 2004;41: 60-66.

30 Thiery JP. Epithelial-mesenchymal transition in development and pathologies. Curr Opin Cell Biol 2003;15: 740-746.

31 Chin D, Boyle GM, Parsons PG, et al. What is transforming growth-beta (TGF- $\beta$ )? Br J Plast Surg 2004;57: 215-221.

32 Tilg H, Diehl AM. Cytokines in alcoholic and nonalcoholic steatohepatitis. N Engl J Med 2000;343: 1467-1476.

33 Osmundsen H, Bremer J, Pedersen JI. Metabolic aspects of peroxisomal beta-oxidation. Biochim Biophys Acta 1991;1085:141-158.

34 Fausto N, Laird AD, Webber EM. Liver regeneration. 2. Role of growth factors and cytokines in hepatic regeneration. FASEB J 1995;9:1527-1536.

35 Schuppan D, Koda M, Bauer M, et al. Fibrosis of liver, pancreas and intestine: common mechanisms and clear targets? Acta Gastroenterol Belg 2000;63: $366-370$.

36 Schuppan D, Krebs A, Bauer M, et al. Hepatitis C and liver fibrosis. Cell Death Differ 2003;10:S59-S67.

37 Lau AH, Thomson AW. Dendritic cells and immune regulation in the liver. Gut 2003;52:307-314.

38 Zimmermann A. Regulation of liver regeneration. Nephrol Dial Transplant 2004;19:6-10. 